\title{
DEVELOPMENT OF LOW COST WIRELESS PLC
}

\author{
Dr.Prof.M.S.Kumbhar \\ Department of Electronics Engineering, \\ R.I.T.Sakhrale, Maharashtra, \\ India
}

\begin{abstract}
Now a days a significant growth in wireless technology has occurred which enabled innovation in the field of wireless technologies. This innovation in wireless technologies leads automation in industrial, residential and commercial sectors. Presently in many industries Programmable logic controller is used to control the entire process automatically without errors and with less human interference. Rapid growth in wireless technology has brought wireless system in existence which removed cabling infrastructure and obtain efficient control. PLCs are used to automate entire process applications in homes and small scale industries. To develop wireless system we need to have a wireless control which is achieved by replacing existing ples with wireless plcs. But it is inconvenient to use wireless PLCs which are costly for small process applications in small scale industries. This paper represents a new method for converting the existing wired plc into wireless plc by configuring wireless module using TMFT software as communication interface between plc and small process, the process used here is called batch process and controlled through HMI.
\end{abstract}

Keywords - PLC, XBEE, TMFT software and HMI

\section{INTRODUCTION}

In the past few decades a lot of research has been carried out in the automaton field. There are various wireless communication protocols has been developed in the past which has resulted into development of automation fied. With the increase of wireless technologies, it is essential to use an appropriate wireless protocol according to application requirement [1]. The implementation of wireless protocols in industrial automation systems is rapidly increasing because of different advantages such as cost effectiveness, Easy placement and installation, small size and portability, scalability feature which provides easy extension, various comfort benefits and feasibility and mobile device connectivity.

It is important to select and implement an appropriate technology according to the desired application requirement. There are different factors such as network topology, network span and transmission time which influence the performance of the system. All wireless protocols are used for different suitable areas of application. The development of particular wireless technology depends on various metrics such as data rate, coverage area, power consumption. scalability, capacity etc.

A Programmable logic controller (PLC) is a device which is used to replace the necessary sequential relay circuits for controlling of relay. We have chosen the Delta make PLC (DVP14SS) in which the ladder diagram is

\author{
Mr.Amar S.Tamboli \\ M.Tech.-II, Department of Electronics Engineering, \\ R.I.T.Sakhrale, Maharashtra, \\ India.
}

designed for the batch process As the plc uses modem for transmitting signals from plc to the process so here we are using Xbee as the communication interface which transmits and receives the signals from the plc to process and viceversa[2][3].Xbee is configured in direct mode by using TMFT software. We have used two Xbee's in the process application. One is for the process side and other is for plc. We have configured both Xbee's to communicate with each other by using TMFT software. The whole process is monitored and controlled through HMI in which the whole process is designed and interfaced with ladder diagram.

The rest of the paper is organized as follows. Need of automation and wireless technology is explained in section 2.Literature survey is presented in section 3. Methodology alongwith proposed system, module programming , simulation and batch process is presented in section 4.Concluding remark is given in section 5 .

\section{NEED OF AUTOMATION AND WIRELESS TECHNOLOGY}

Now a days automation is used in various control systems such as numerical control, programmable logic control and other industrial control systems. Use of Automation is incorporating widely in other controlling applications of information technology (such as computer-aided technologies CAD, CAM), to control industrial mechanism,hardware and processes, Automation is reducing the need of human intervention. In the industrial controlling processes, automation is a step beyond mechanization. Whereas mechanization provides human being as operators alongwith machinery provided by mechanization to support them with the physical requirements of work. Automation greatly reduces the need of human efforts as well as mental requirements. Processes and different controlling systems can also be automated. Currently there are many different tasks for humans in industrial processes which lie beyond the scope of automation. Human-level pattern recognition, language recognition, and language production ability are well beyond the potential of modern mechanical and computer systems. Tasks requiring subjective evaluation or composition of complex sensory data, such as scents and sounds, as well as high-level exercises such as strategic planning, presently require human beings which are skilled and expertise. In many times, the use of human beings is more costly and might be defective than mechanical appearance even where automation of industrial processes is possible. Specific accustomed computers, referred to as programmable logic controllers (PLCs), are very often used to control and moitor the flow of inputs from (physical) sensors and events with the flow of outputs to actuators and 


\section{International Journal of Engineering Applied Sciences and Technology, 2019 Vol. 4, Issue 8, ISSN No. 2455-2143, Pages 135-143 \\ Published Online December 2019 in IJEAST (http://www.ijeast.com)}

events[4].This implies accurate controlled actions that allow a fixed control of almost any industrial processes and tasks.

Human-machine interfaces (HMI) formerly known as man-machine interfaces, are usually utilized to communicate with PLCs and other computers, for monitoring and controlling different parameters such as temperature, pressure or any other parameter for controlling further response or process. Service personnel who monitor and control these interfaces are often called as stationary engineers. Presently, for fabricating or processing industries, the intention of automation has been displaced from increasing productivity and reducing expenses, to broader issues, such as increasing quality and tolerance in the manufacturing process. The prime focus of automation in past was simply to increase productivity and reduce expenses which was noticed to be short-sighted, because for it there was necessary to provide a skilled manpower which could make repairs and handle the machinery properly. Moreover, the initial cost of automation was high and often could not be recovered,while at the same time the old manufacturing processes were replaced by the new automation processes. Automation is now often utilized primarily to increase quality in the manufacturing process, where automation can reduce maintenance in large scale and also increase quality significantly[5].

Wireless telecommunication is the transport or exchange of information and messages between two or more units that are not physically connected with each other. Distances may be short, such as a few meters like television remote control, or as far as thousands or even millions of kilometers for deep-space radio communications. It includes various types of fixed, mobile, and portable two-way radios, cellular telephones, personal digital assistants (PDAs) and wireless networks. Other examples of wireless technology include GPS units, garage door openers, wireless computer mice, headphones, radio receivers, satellite television, broadcast television and cordless telephones. Wireless operations grants services, such as long range communications, that are impossible or inconvient to implement with the use of wires. The following situations justify the use of wireless technology:

1. To span a distance beyond the capabilities of typical cabling.

2. To provide a backup communications link in case of normal network failure.

3. To link portable or temporary workstations

4. To overcome situations where normal cabling is difficult or financially impractical.

5. To remotely connect mobile users or networks.

Wireless communications can be through:

1. Radio Frequency Communication

2. Microwave communication, for example long-range line-of-sight via highly directional antennas, or short-range communication.

3. Infrared (IR) short-range communication, for example from consumer IR devices such as remote controls or via Infrared Data Association (IrDA).

Hence presently for fabricating or processing industries automation is required for increasing quality and tolerance in the manuacturing process.

\section{LITERATURE SURVEY}

There are four protocol standards for wireless communications with low power consumption. These four protocols are Bluetooth (over IEEE 802.15.1), ultrawideband ( UWB.over IEEE 802.15.3), ZigBee (over IEEE 802.15.4) and Wi-Fi (over IEEE 802.11).In the industrial automation system for interconnection, It is required to combine different sensors, controllers, machines and hardware.There are different network types for use in automation such as control area network ( CAN ), Process fieldbus (Profibus ), Modbus etc.on the other hand, to access networks and services without wires or cables, wireless communication is a rapidly developing technology to provide the flexibility and mobility.

Bluetooth is used for short range devices to replace cables for computer peripherals such as mice,keyboards and printers based on a wireless radio system. There are two connectivity topologies in Bluetooth : the piconet and scatternet.A Piconet is a wireless personal area network created by a Bluetooth device serving as a master in the piconet and one or more Bluetooth devices serving as slaves.A scatternet is a collection of operational Bluetooth piconets overlapping in time and space[6].

Now a days UWB is used as an indoor short-range highspeed wireless communication.The most useful characteristic of UWB is that its bandwidth is upto 480 Mbps which is required to satisfy multimedia applications such as audio and video delivery in small networking.It also acts as a wireless cable replacement of high speed serial bus such as USB 2.0 and IDEE 1394.

Zigbee over IEEE 802.15.4 is used for supporting simple devices that consume low power and operates in the range of 10m.Zigbee operates on the IEEE 802.15.4 specification and For wireless technology[7][8].Zigbee is an open global standard which is designed to use low power radio signals for personal area network. The communication standard of Zigbee defines physical and Media Access Control layers to handle different devices at low data rates. The zigbee operates in the worldwide at the following frequencies:2.400-2.484GHz , 902-928 MHz and 868.0868.6 MHz.Because of scalable network,long battery life and easy to monitor and control different appliances from remote places,Zigbee is most suitable for designing of automation system[9][10].

Wireless fidelity ( Wi-Fi) is wireless networking technology which uses radio waves to provide wireless high speed internet and network connections. Wireless fidelity includes IEEE 802.11 family of standards for Wireless local area networks ( WLAN ) .Desktops and laptops,smartphones and tablets, digital cameras,printers can use Wi-Fi technologies. The different versions of Wi-Fi are defined by various IEEE 802.11 protocol standards, with different radio technologies determining the ranges and speeds that may be accomplished.

A PLC is a controller used for automation of processes in industries such as control of machinery on factory assembly lines.Now a days PLC is most popularly used in the industry because of its flexibility,reliability,ease of programming,ease of hardware usage and cost 


\section{International Journal of Engineering Applied Sciences and Technology, 2019 \\ Vol. 4, Issue 8, ISSN No. 2455-2143, Pages 135-143 \\ Published Online December 2019 in IJEAST (http://www.ijeast.com)}

effectiveness[11].Programmable Logic Controllers are used for continuously recoding or observing the input values from sensors and devices and produces the controlling outputs for the operation of processes or actuators based on the program.

Each PLC system built up of CPU module, Power supply module and one or more I/O module. CPU Module consists of central processor and its memory. The processor performs all necessary computations and process the data by accepting the inputs and produces the appropriate outputs. The power supply module provides the required power to entire system or process by converting the available $\mathrm{AC}$ power to $\mathrm{DC}$ power required for the CPU and I/O modules of the PLC.The input and output modules of the PLC are used to attach the sensors, devices and actuators to the system for sensing the different parameters like temperature, pressure and flow etc. These I/O modules are of digital or analog types.

A programmable logic controller, PLC, or programmable controller is a small computer used for automation of real-world processes. The PLC usually uses a microprocessor. The program can continually manage complex sequencing and is written by skilled engineers. The program is stored in battery-backed memory and/or EEPROMs. The main difference from other computers is the special I/O arrangements. This connects the PLC to sensors and actuators. The I/O provisions may be construct into a simple PLC, or the PLC may have external $\mathrm{I} / \mathrm{O}$ modules connected to a proprietary computer network that put into the PLC.

A single PLC can be arranged to restore 1000s of relays. Programmable controllers were initially used by the automotive manufacturing industry. The functionality of the PLC has developed over the years to include typically relay control, sophisticated motion controller, process control, distributed control systems and complex networking. However, it should be noted that PLCs are more expensive, contain everything needed to handle high power loads right out of the box. In addition, a micro controller- based would not have the flexibility of infield programmability of a PLC. So PLCs are more popularly used in manufacturing or mass production lines.

\subsection{Architecture of PLC}

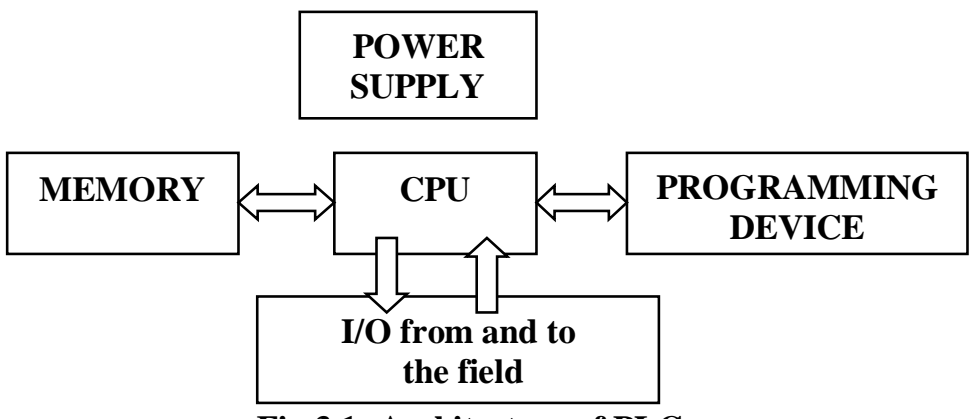

The central processing unit of the architecture is considered to be the heart of the programmable logic controller. We can program the PLC in the field itself. This can be done using a programming device which can be a hand held computer or a personal computer. The communication with the system will be a serial communication using a RS-232 or RS-485 cable. The control program is stored in memory. The external power supply module satisfies the need of all the modules. It directly controls the outputs taking into consideration of the various inputs. Programmable logic controllers (PLC) have been evolved out from Relay logic circuits (RLC). The relay logic circuits are hard wired. These RLC's have many drawbacks such as bulky circuits, inflexibility and troubleshooting is very difficult. The PLC is the ultimate solution to overcome all these problems.

\subsection{Basic Parts of PLC}

\section{CPU (Central Processing Unit)}

The CPU is the heart of the PLC system. The CPU is a microprocessor-based control system that put backs central relays, counters, timers and sequencers. A processor come into view only once in a PLC and it can be either a one-bit or a word processor. One bit processors are sufficient for dealing with logic operations. PLCs with word processors are used when processing text and numerical data, calculations, gauging, controlling and recording, as well as the simple processing of signals in binary code are required. The basic principle of it is as follows.

The CPU accepts (reads) input data from various sensing devices (sensors) executes the user program from memory and sends appropriate output commands to control devices. To produce the low-level voltage to be used by processor and I/O modules, a direct current (DC) power source is required. This power supply can be arranged in the CPU unit itself or may be a separately arranged unit, depending on the PLC system manufactures. The CPU contains various electrical parts and boxes for connecting the cables that go to the other units as well as to operational key switches.

Typical operation key switch positions are:-

1) Off: system cannot be run or programmed.

2) Run: allow the system to run, but no program modifications can be made.

3) Program: disables output and allows creating modifying, and deleting of programs.

\section{Input \& Output Modules of PLC}

Input/output modules are used for interfacing between input devices (e.g. start and stop push buttons, sensors, limited switch, selector switch) and microprocessor. And also used for interfacing between output devices (e.g. electrical heater, lights, solenoid valve, relays, buzzer, fan) and microprocessor. The input information is transfer to the processor through input module and output information from the processor to load is transformed through output module.

The input/output module is used for providing the separation between input devices and process and also to provide separation from processor to output devices. Optocoupler is used for separation. Input devices like push buttons, limited switches, sensors and selector switches are hard-wired to terminals on the input modules. Output devices like small motor, motor starters, solenoid valve, and indicator lights are hard wired to the output modules. 


\section{International Journal of Engineering Applied Sciences and Technology, 2019 \\ Vol. 4, Issue 8, ISSN No. 2455-2143, Pages 135-143 \\ Published Online December 2019 in IJEAST (http://www.ijeast.com)}

Input interface modules accept signals from the machine or process devices (e.g. $220 \mathrm{~V} \mathrm{AC)}$ and convert them into signals (e.g. 5VDC) that can be used by the controller, or processor output interface modules transmits controller or processor signals (e.g. 5VDC) into external signals (e.g. 220 $\mathrm{v}$ AC) used to control the machine or process. The input modules perform four basic takes in PLC system.

1) They sense when signal is received from the sensor on the input of the machine,

2) They convert the input voltage to the correct voltage level for the PLC

3) They isolate PLC from fluctuation in the input signal voltage or current.

4) At end they send the signal to the PLC.

\section{PLC Output Module}

Output module is use for interfacing between output load and processor. The output module has a function similar to that of the input modules except in reverse order.
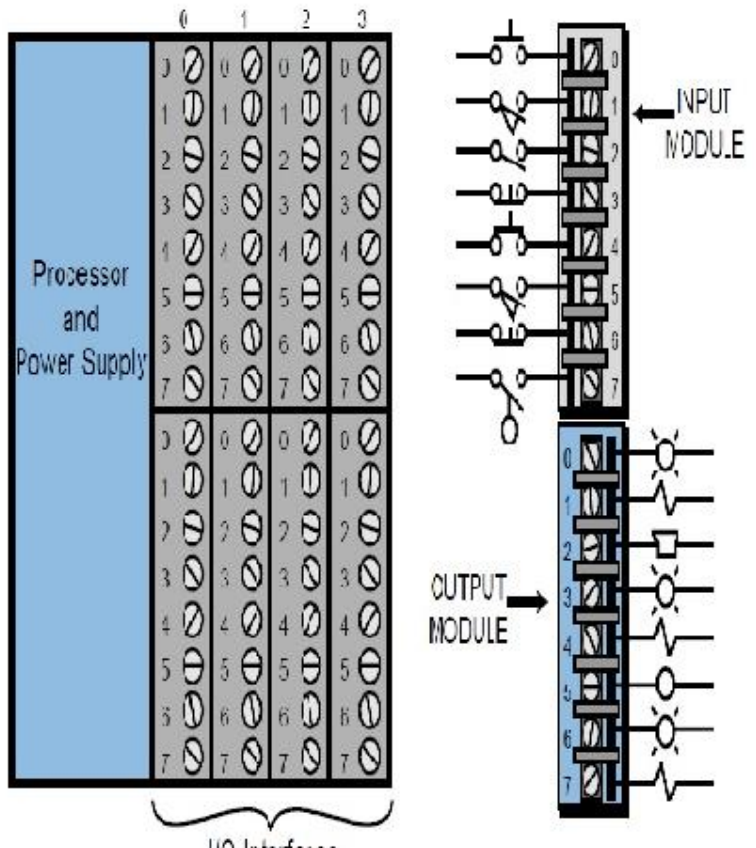

Fig 3.2: Input \& Output Modules of PLC

\section{Programming device (Keyboard Monitor)}

Keyboard and monitor is used for programming a PLC. The data is entered in the PLC processor with the help of Keyboard in the form of ladder diagram. This ladder diagram can be seen on the monitor screen. The programmer can communicate with PLC processor with help of programming devices. The programming unit communicates with processor if PLC via a serial or parallel data communication is linked. The personal computer can be used for programming of PLC if it has required software.

\subsection{Zigbee Transceiver}

To use PLC wireless module or a wireless modem for wireless exchange is expensive so ZigBee module can be used along with PLC to transmit data to and fro from the process[12]. The zigbee module is interfaced to a host device through a logic-level asynchronous serial port. Through its serial port, the module can communicate with any logic and voltage compatible UART or through a level translator to any serial device (For example: RS-232 or USB interface board). Zigbee can be interfaced with a micro controller or a PC using serial port with the help of appropriate level conversion.

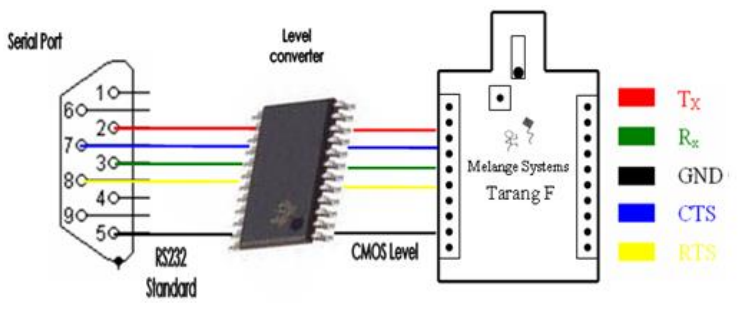

Fig.3.3: Serial Interface

To establish a successful serial communication with the module, serial parameters need to be configured properly in the module and host side. Both the module and PC settings can be viewed and set using AT command set through popular terminal applications like 'HyperTerminal' [13].

Thus for better wireless communication we can use Zigbee and PLC in small scale industrial applications or home based small applications.

\subsection{Proposed System}

\section{METHODOLOGY}

The block diagram of the proposed work is shown in Figure 4.1. In the proposed system we are replacing existing small scale industries based wired PLC system into wireless PLC System by configuring zigbee wireless module as communication interface between PLC and process. In this proposed system we are using PLC to automate the small process and also to monitor it. Now a days Programmable Logic Controller is used in many industries to control the full method mechanically with less human intervention and to avoid errors. With the rapid climb in technology, wireless instrumentation has came into existence to avoid cabling infrastructure and to obtain efficient control. PLCs are also used in small scale industries to automate small process applications. We are using the Delta make PLC(DVP14SS), and ladder diagram is designed in it for desired application. As the wireless plcs use modem for transmitting signals from plc to the process, We are using TARANG Zigbee wireless module as the communication interface which is used for transmitting and receiving the signals from the plc to process and vice-versa.

In our proposed system we are proposing a process in which level the main parameter is to be controlled and the setpoint for this parameter is given by the operator/user in the HMI interface and also we are going to monitor and control mixing of two liquids. The process is in one side and the plc on the other side will transfer and receives the signals through the wireless modules placed in between them. The process is initiated by the user from the HMI interface and can be monitored and controlled by observing the flow of the process designed which is shown in the block diagram.

The PLCs provide analog and digital series input/output that can be used to control the field devices. 


\section{International Journal of Engineering Applied Sciences and Technology, 2019 \\ Vol. 4, Issue 8, ISSN No. 2455-2143, Pages 135-143 \\ Published Online December 2019 in IJEAST (http://www.ijeast.com)}

For the PLC to be made to regulate knowledge wirelessly, a wireless interface is needed. The messages from the controller are sent to PLCs through the RF transceivers. Thus, two RF transceiver circuits have to be developed such that they are able to communicate with each other which is shown in Figure 4.2

The set point for level control is given in the PLC/HMI by the user. Then according to control algorithm written in PLC, the control signals from output module of the controller is given to ZigBee 'A', which acts as a transmitter. Wireless exchange of data takes place and the signal reaches to ZigBee 'B' which acts as a receiver on the process side. These signals from ZigBee ' $B$ ' reaches the field/process through relays on the process side and actuate the actuators.

The sensors (level) sense the corresponding signals and the level of the process is given to ZigBee ' $\mathrm{B}$ ' which now acts as a transmitter. Wireless exchange of data takes place and the signal reaches to ZigBee 'A' which now acts as a receiver on the control room side. The signals from ZigBee ' $A$ ' reaches to the controller through the relays to the PLC input module and this continues till the set point (given by the user) is reached and the level is maintained. Thus a low cost wireless PLC system can be designed and used for remote monitoring throughd HMI[14].

$\mathbf{T}_{\mathbf{x}}$

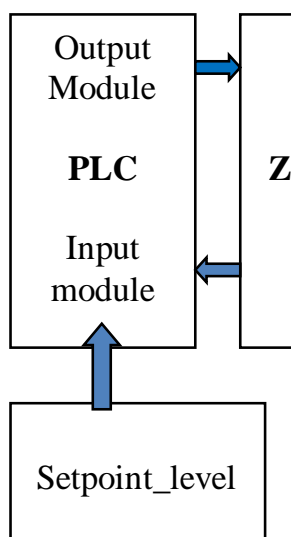

Figure.4.1. Block diagram of proposed system.

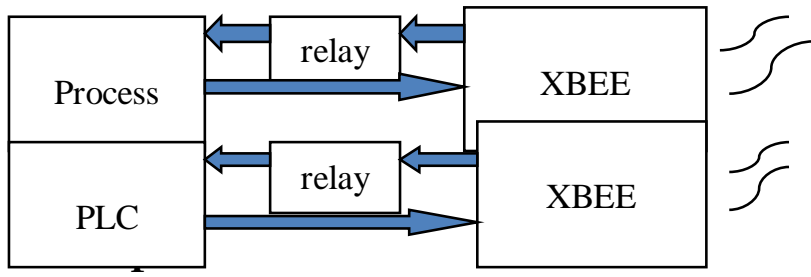

igure4..2.RF Transceiver Diagram.

\subsection{Module Programming}

Step 1: Open TMFT Software

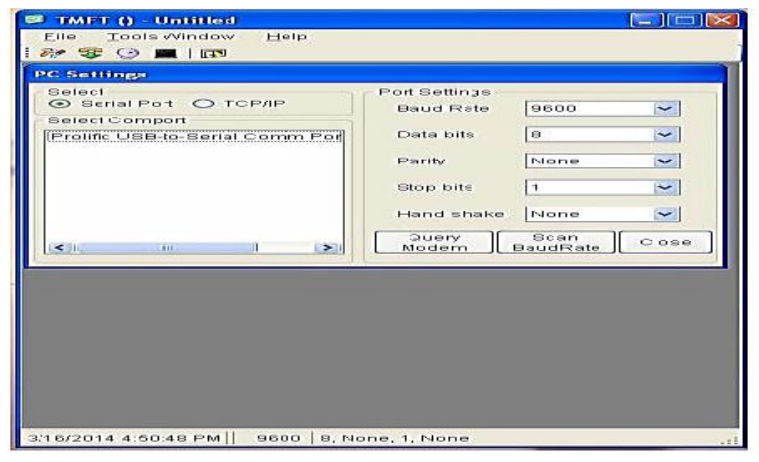

Step 2: Connect the module to the Serial/USB Port.

Step 3 : Choose the appropriate Port and serial parameters in terminal software \& press query modem.

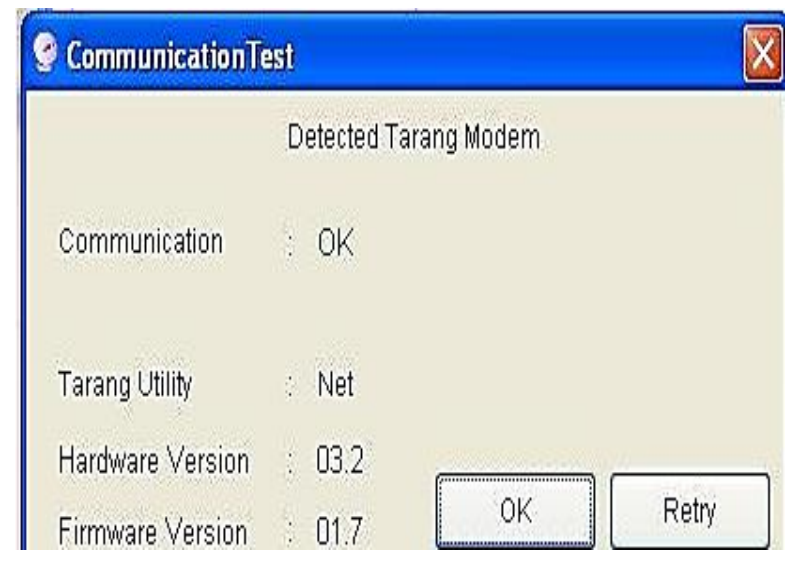

Step 4: For setting I/O pins as input and output the following steps should be followed

Step 5: Enter the command mode with ' +++ ' Response from modem should be ok.

Step 6: Enable the desired I/O pin as input with command ATIDxx. For configuring it to

Digital I/O input, send command as ATID02.

Response from module should be 'OK'. 
International Journal of Engineering Applied Sciences and Technology, 2019

Vol. 4, Issue 8, ISSN No. 2455-2143, Pages 135-143

Published Online December 2019 in IJEAST (http://www.ijeast.com)

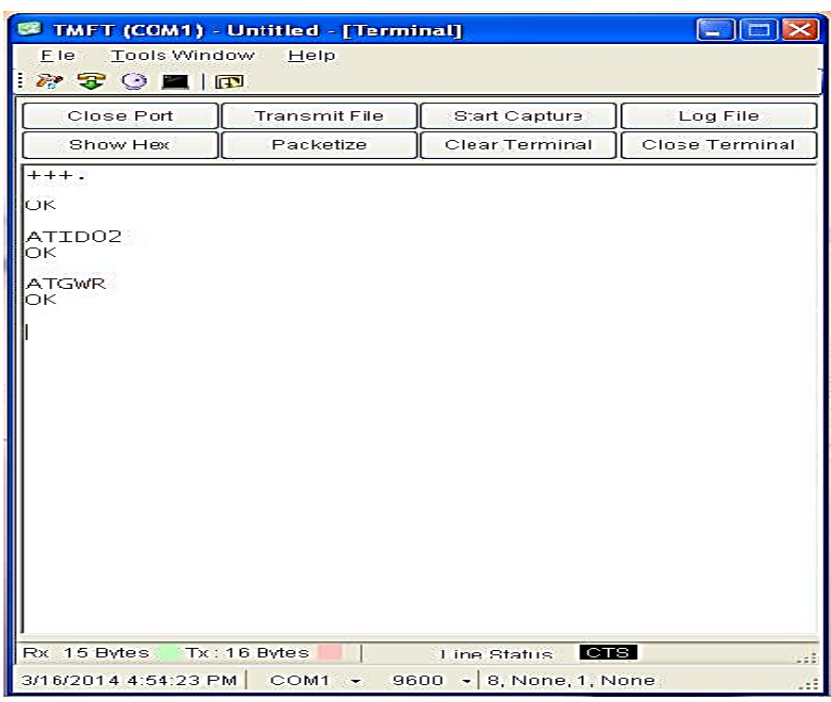

Step 7: Write these parameters to memory with 'ATGWR' command.

Step 8: Follow the same steps for configuring I/O pins to INPUT.

Step 9: Exit command mode with 'ATGEX' command.

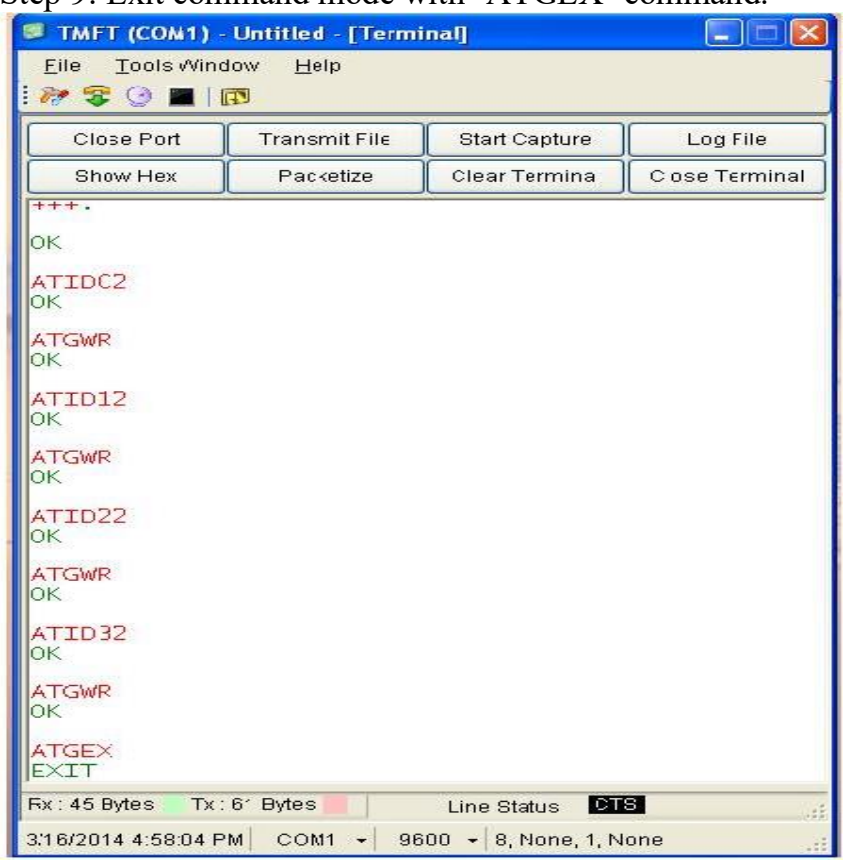

Step 10: Enable the desired I/O pin as input with command ATIDxx.For configuring it to Digital $\mathrm{I} / \mathrm{O}$ input, send command as ATID40. Response from module should be 'OK'

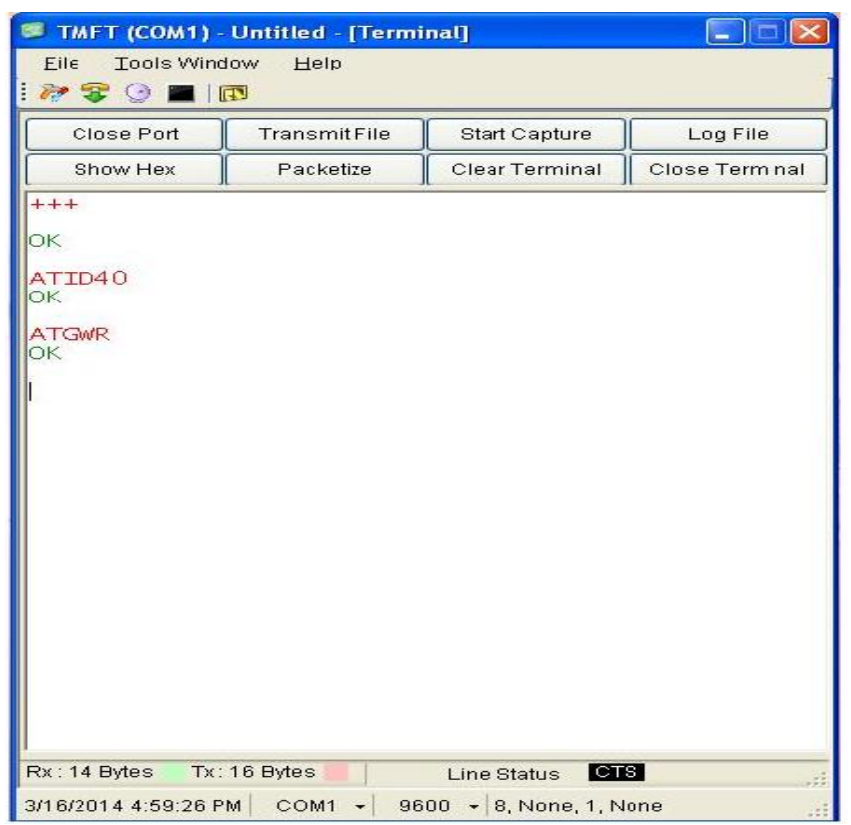

Follow the same steps for configuring module I/O pins to output.

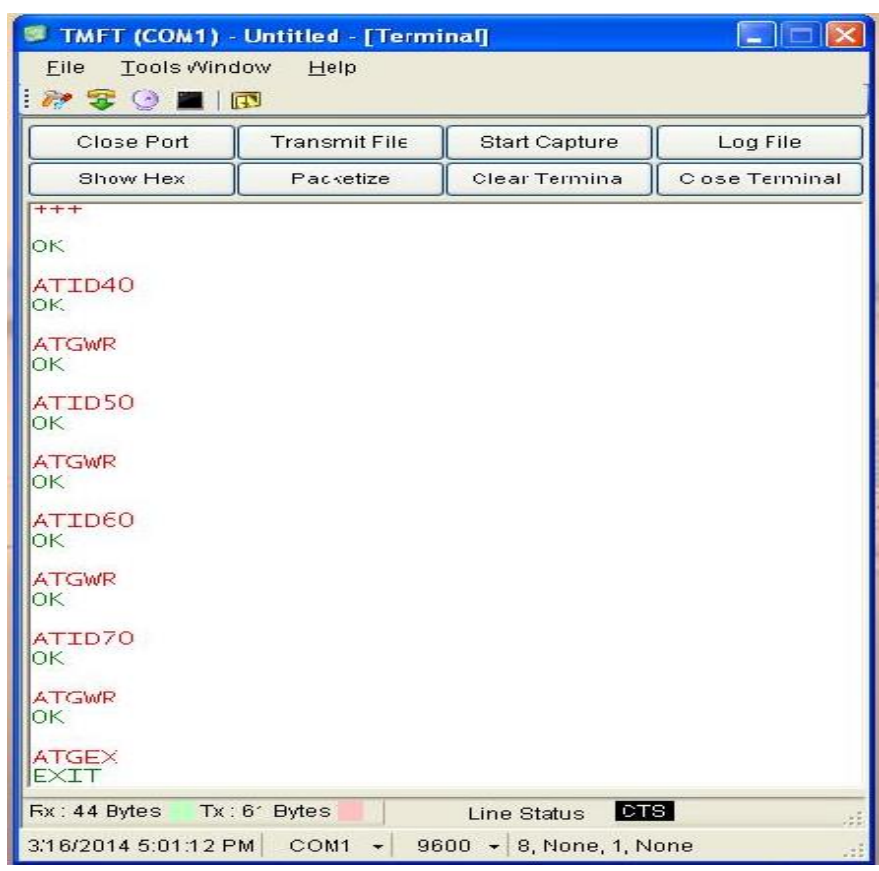

This is the procedure for configuring ZigBee for control side. Follow the same steps for configuring another module I/O pins for field work.

\subsection{Simulation}

Proposed Work for batch process is developed in Delta WPLSoft/SIMULINK. Simulation model for mixing of two liquids in proportion is shown in Figure 3. Some applications needs specific quantities of raw materials to be combined in specific ways for particular durations to provide an intermediate or end result. A batch process performs list of actions in a sequence. It executes a series of all non-interactive actions at a one time. Once a batch 
Published Online December 2019 in IJEAST (http://www.ijeast.com)

process job begins, it continues until it is done. Batch processes are generally used to produce a relatively low to intermediate quantity of product per year (a few pounds to millions of pounds). One example is that the production of adhesives and glues, which normally require the mixing of raw materials in a heated vessel for a period of time to form a quantity of end product. Other necessary examples are the production of food, beverages and medicine.

Batch Process components are Process Tank 1(for mixing of two liquids in proportion), Tank A, Tank B, Stirrer, DC Motor, Solenoid Valve 1, Solenoid Valve 2, Solenoid Valve 3 and high level float switch.

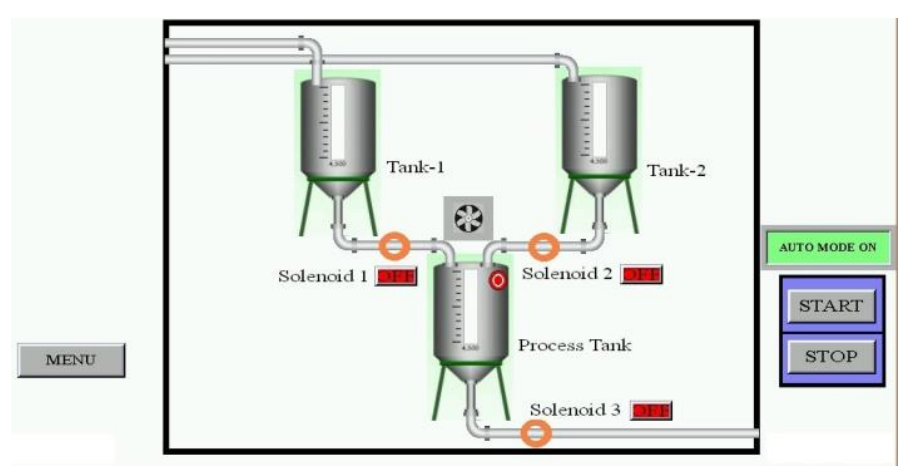

Fig :4.3.1 Simulation Model For Mixing of Two Liquids in Proportion.
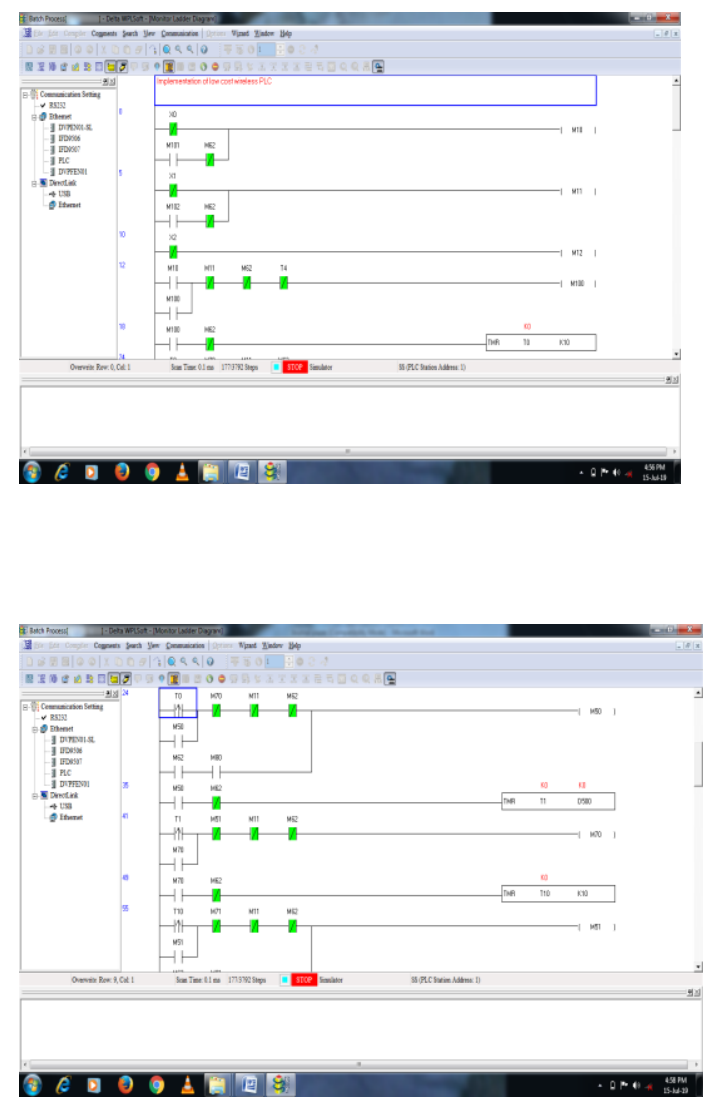
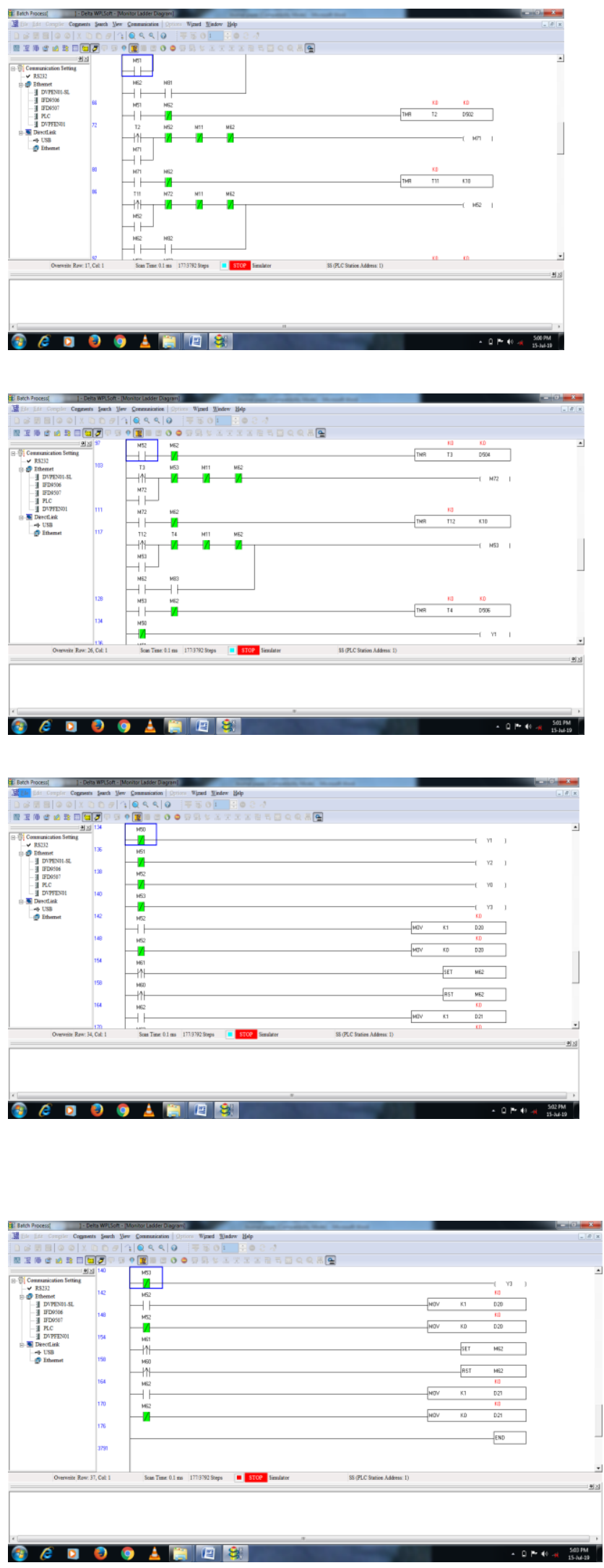

Fig :4.3.2 Simulation for mixing of two liquids in proportion. 


\section{International Journal of Engineering Applied Sciences and Technology, 2019 Vol. 4, Issue 8, ISSN No. 2455-2143, Pages 135-143 \\ Published Online December 2019 in IJEAST (http://www.ijeast.com)}

\subsection{Process Description}

The BATCH PROCESS setup is placed in a remote area (field/process site) and is controlled from the control room wirelessly using ZigBee where PLC, HMI and the control panel are placed. The process is controlled through HMI\&PLC in auto mode and can also be controlled in manual mode. The process can be controlled and monitored directly from HMI and also from the control panel.

The control panel controls the field or process site. The main components are ZigBee and PLC which control the whole process. Zigbee is used as the source and which is used in direct mode. PLC is employed to manage and monitor the batch process.

When the START button is pressed by the user then batch process is started. Then according to control algorithm written in PLC, the control signals from output module of the controller is given to ZigBee 'A', which acts as a transmitter. The first solenoid valve 1 is $\mathrm{ON}$ for some time from TANK A and then solenoid valve 2 is on for some time from TANK B. At that time if process tank becomes overflow then either solenoid valve 1or solenoid valve 2 is off and avoid overflow. After this agitator is on which mix the two liquids uniformly. As soon as agitator is off we get uniform mixed liquid and solenoid valve 3 is on and we get the output. At this operation ZigBee on process side acts as a receiver and ZigBee on control side acts as a transmitter

Now if the tank level of TANK A, TANK B or PROCESS tank becomes high the signal is received by ZigBee on process side and sends this signal to control side ZigBee which monitor on input section of PLC and HMI. At this operation ZigBee on control side acts as a receiver and ZigBee on process acts as a transmitter.

\section{CONCLUSION}

Batch Process is a very important and a widely used process in today's Industries. Even though it is a widely used process, it is yet to be controlled wirelessly. The drawbacks faced by the Industries are dealing with extra cablings and its faults. We have tried to eliminate the use of cables for transmission and overcome a major drawback in the setup. We achieve the task of monitoring as well as controlling the Batch Process from a remote location via a control panel, PLC program as well as HMI. The parameter controlled in a closed loop process is Level.

\section{ACKNOWLEDGMENT}

I would like to thank Dr.M.S.Kumbhar for inspiring me to write this research paper. I am expressing my gratitude to everyone who supported me for writing this research paper. I am thankful for their guidance and invaluably advice during this work. I am sincerely grateful to them for sharing their truthful and informative views on a number of issues related to this paper.

\section{REFERENCES}

1) Jin Lee,Y.,Wei S., and Chung S.(2007). "A Comparative Study of Wireless protocols: Bluetooth,UWB,Zigbee and Wi-Fi",The33 ${ }^{\text {rd }}$ Annual Conference of the IEEE Industrial Electronics Society, (pp.46-51)

2) Shuang G.,Yao H., and Xin L.(2009). “A zigbee-based home automation system", Consumer Electronics, IEEE Transactions on , vol.55, no.2, (pp.422-430).

3) Yang L., Maorong J., and Zhenru G.(2009). "Design of Home Automation System based on ZigBee Wireless Sensor Network", Information Science and Engineering (ICISE), 2009 1st International Conference, (pp.2610- 2613).

4) Xiaolong L.,Munigala S., and Zeng Q.(2010). “Design and Implementation of a Wireless Programmable Logic Controller System". International Conference on Electrical and Control Engineering in IEEE,(pp.3138-3141).

5) Wins B, Direrot P.(2016). "Design Of Network architecture For Home Energy System Based On Zigbee Using Remote Control", Available at:www.iccce.co.in/Papers/ICCCECS678.pdf

6) Baker N.,(2005). "ZigBee and Bluetooth: Strengths and weaknesses for industrial applications", Proceedings of IEEE Computing and Control Engineering, vol. 16, no. 2, (pp 20-25).

7) Baviskar J., Mulla A., Baviskar A., Panchal N., and Makwana R.(2014). "Implementation of 802.15.4 for Designing of Home Automation and Power Monitoring System" IEEE Students Conference on Electrical,Electronics and Computer Science,(pp.978982)

8) Baviskar J.,Mulla .,Upadhye M.,Desai J., and Bhovad A.(2015). "Performance Analysis of Zigbee based Real Time Home Automation System",International Conference on Communication,Information and Computing Technology of IEEE,(pp.978-983).

9) Francesco M.,Anastasi G.,Conti M.,Das S.,and Neri V.(2011)."Reliability and Energy-efficiency in IEEE 802.15.4/ZigBee Sensor Networks:An Adaptive and Cross-layer Approach", IEEE journal on selected areas in communications, vol. 29 , no. 8 .

10) Anastasi G.,Conti M.,Francesco M.,and Neri V.(2013).“Reliability and Energy Efficiency in Multihop IEEE 802.15.4/ZigBee Wireless Sensor Networks",IEEE 11th Malaysia International Conference on Communications , Kuala Lumpur, Malaysia.

11) Guo H.,hua C.,Hao F., shuanGan W.,and Chen C.(2010)."Research of wireless Communication Between PLC and Computer based on nRF2401", International conference on Communications and Mobile Computing, Volume 3.

12) Cuomo F., Luna S.,Monaco U.,and Melodia T.(2007)."Routing in ZigBee: Benefits from exploiting the IEEE 802.15.4 association tree", IEEE Communications Society subject matter experts for publication in the ICC proceedings. 
13) Egan D.(2005).“"The emergence of ZigBee in building automation and industrial control," Computing and Control Engineering Journal, vol. 16, no. 2, (pp. 1419).

14) Bhuiya K.,Kintali A.,Dhwani P.,and Kilaru L.(2013). "Wireless Control and monitoring using PLC and SCADA" International Journal of Scientific and Research Publications, VOL. 3, Issue 9, (pp.112-115). 